\title{
Intrauterine Temporomandibular Joint Dislocation: Prenatal Sonographic Evaluation
}

\author{
Ahmet Said Çil; Murat Bozkurt; and Duygu Kara Bozkurt
}

\begin{abstract}
Congenital temporomandibular joint (TMJ) diseases are very rare disorders and are usually diagnosed in childhood. Developmental disorders of the TMJ such as hypoplasia, hyperplasia, and aplasia of the TMJ compartments are characterized by TMJ dysfunction. In childhood, these patients experience recurrent dislocation, pain, and malocclusion. We present the case of a 25-week fetus with unilateral TMJ dislocation with fluid retention in the joint diagnosed by ultrasonography. To the best of our knowledge, this is the first case of TMJ dislocation diagnosed by ultrasonographic evaluation during the prenatal period.
\end{abstract}

Keywords: Mandibular condylar hypoplasia; Prenatal diagnosis; Prenatal ultrasonography

Temporomandibular joint dislocation

Corresponding Author:

Corresponding Author:

Murat Bozkurt, MD

Kafkas Üniversitesi Sağlık Araştırma ve Uygulama Hastanesi

Kadın Hastalıkları ve Doğum Kliniği

Kars, Turkey

Tel: $+905322279072 ;+905056330044$

Fax: 04742251430

E-Mail: jindrmb@yahoo.com

Received: January 16, 2013

Revised: April 17,2013

Accepted: May I, 2013

doi: $10.3121 / \mathrm{cmr} .2013 .1148$
$\mathrm{T}$

he temporomandibular joint (TMJ) is typically completely differentiated in the $25^{\text {th }}$ to $26^{\text {th }}$ gestational week. ${ }^{1}$ Several studies have been reported about TMJ development through gross specimen or microscopic examinations of postmortem embryos. There have been no scientific studies about sonographic prenatal evaluation of the TMJ development and disorders.

We searched the MEDLINE database using a combination of keywords including 'temporomandibular joint,' 'prenatal diagnosis,' and 'ultrasound'; however, no study or case report was retrieved. Further, only a few case reports have been published regarding childhood TMJ dislocation., ${ }^{2,3}$ We report, to the best of our knowledge, the first case of TMJ dislocation that was diagnosed incidentally by real-time ultrasonographic evaluation during the prenatal period.

\section{Case Report}

A healthy woman, aged 27 years, in the $25^{\text {th }}$ week of her first pregnancy, was referred to our clinic for a genetic sonogram, which was performed using a LOGIQ 9 ultrasound system (GE Healthcare) with linear $(7.5-5 \mathrm{MHz})$ and convex $(3.5 \mathrm{MHz})$ transducers. In the right TMJ, fluid retention was observed in the joint capsule. The mandibular condyle was in the normal position when the mouth was closed (figure 1). As the jaw opened, the mandibular condyle was displaced to an anterolateral position and was not reduced in the articular fossa during the examination (figure 2A). The articular disk was not clearly visible on sonographic examination. The left mandibular condyle was in the normal position without fluid retention inside the joint (figure $2 \mathrm{~B}$ ). We also measured the anterior/posterior (AP) and lateral diameters of the bilateral mandibular condyles. The right AP diameter of the mandibular condyle measured $3 \mathrm{~mm}$, and the lateral diameter, measured $4.2 \mathrm{~mm}$; whereas the left AP diameter of the mandibular condyle measured $3.6 \mathrm{~mm}$, and the lateral diameter measured $4.6 \mathrm{~mm}$. Furthermore, we observed that the right mandibular condyle was smaller than the 


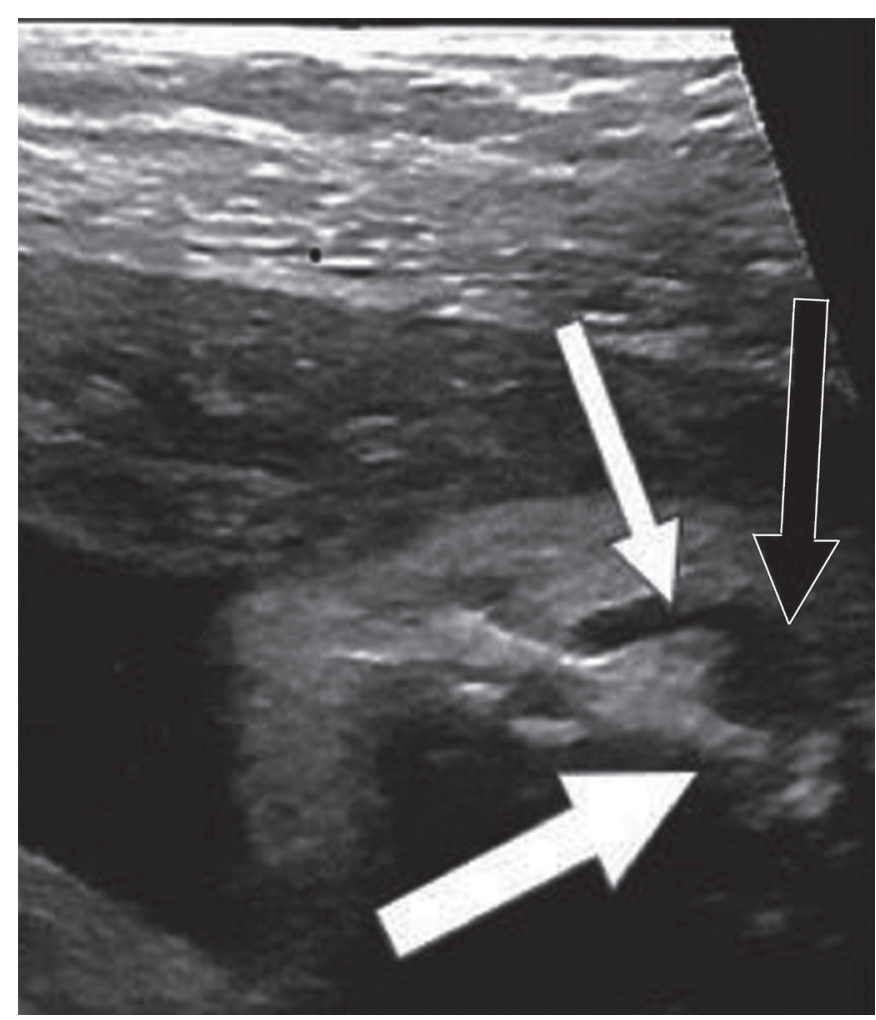

Figure 1: Sagittal view of the right TMJ; mouth was closed and right mandibular condyle was in the normal position (large white arrow) with fluid retention in the joint (black arrow).

left, and the right articular fossa was smaller than the left. This situation led us to believe that the dislocation could be due to hypoplasia. During the $35^{\text {th }}$ week of gestation, we again evaluated the fetus with sonographic examination. Although the right condyle was normally positioned, fluid retention was observed in the right TMJ, and the joint capsule appearance was thick and edematous. The mandibular condyle was hypoechoic with irregular contour (figure 3). These findings suggested that the deformation of the joint structure could be caused by repetitive dislocation of the right TMJ. During the examination, we were unable to evaluate the left TMJ because of the position of the fetus.

Three months after the birth of the child, we reevaluated the infant. On physical examination, minimal facial asymmetry and malocclusion was observed (figure 4). Other systemic physical examination and laboratory findings were within normal limits. The mother did not describe any problems with TMJ dislocation during breastfeeding or any locking of the jaw. The infant's father reported a history of recurrent TMJ dislocation and locking of the jaw, especially with yawning. The infant and father were both referred to the oral and maxillofacial surgery department of an another hospital for detailed examination and treatment, as this type of unit has not yet been established in our hospital.

\section{Discussion}

Primary TMJ diseases comprising congenital and developmental alterations of the TMJ include hyperplasia, hypoplasia, and aplasia of the joint structures. ${ }^{1}$ During TMJ development, the articular fossa is the first structure to evolve, usually in the $7^{\text {th }}$ to $8^{\text {th }}$ gestational weeks. The articular fossa begins to ossify between the $10^{\text {th }}$ and $11^{\text {th }}$ gestational weeks. The development process of the condylar cartilage occurs between the $10^{\text {th }}$ and $11^{\text {th }}$ gestational weeks from the accumulation of the mesenchymal cells laterally to Meckel's cartilage. During development, apical endochondral ossification proceeds and creates a bony fusion with the body of the mandible. After the $7^{\text {th }}$ to $8^{\text {th }}$ weeks of gestation, the articular disk can be identified as a horizontal concentration
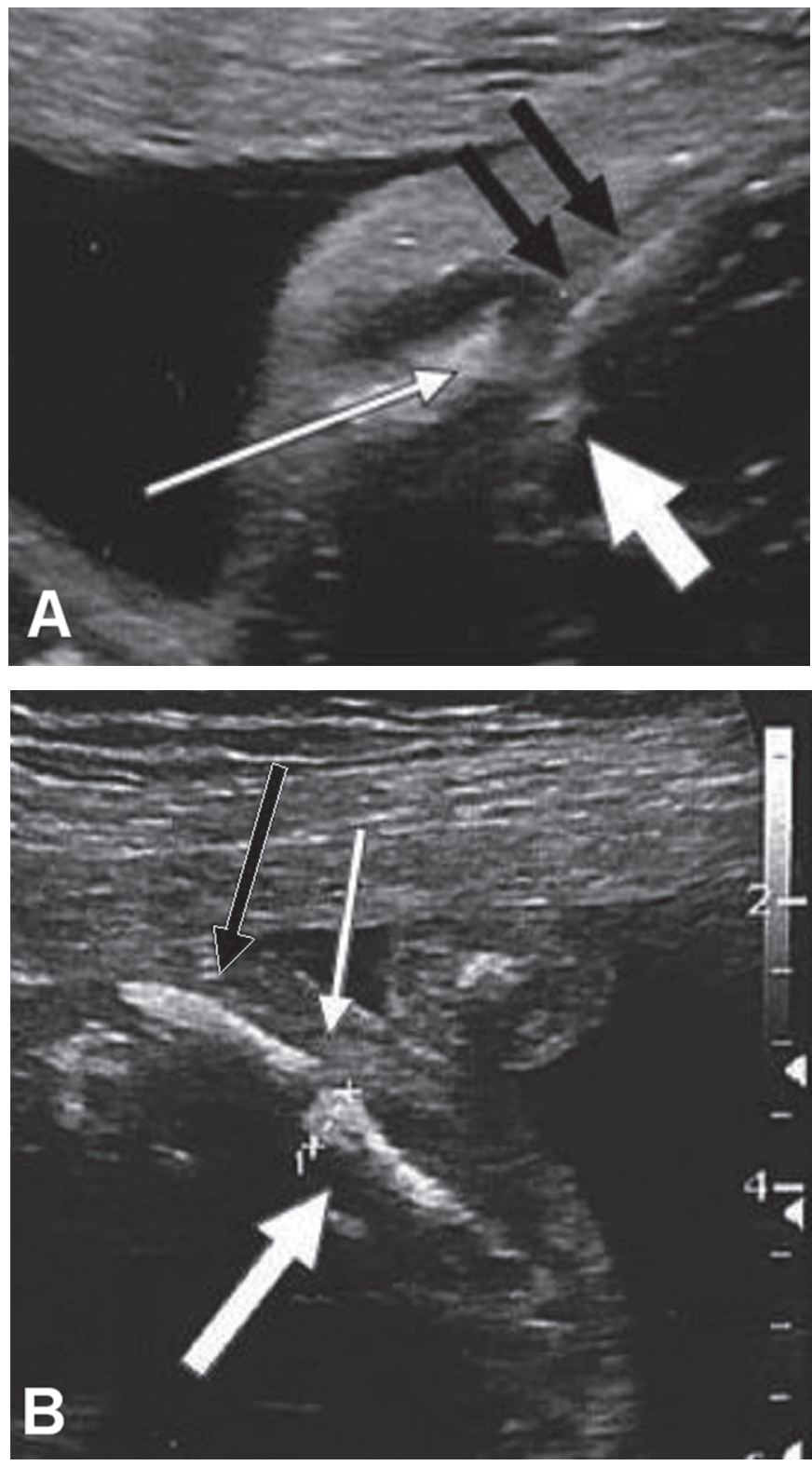

Figure 2: $(A)$ Coronal oblique view of the right TMJ; mandibular condyle was dislocated to the anterolateral (thin white arrow) position, the temporal bone border (black arrows) was not continuous with the mandibular ramus. The temporal fossa viewed shallow (large white arrow). (B) Transverse oblique view of the left TMJ; the mandibular condyle (large white arrow) is normally positioned in relation to the articular fossa (thin white arrow). The temporal bone border (black arrow) is continuous with the mandibular ramus. 


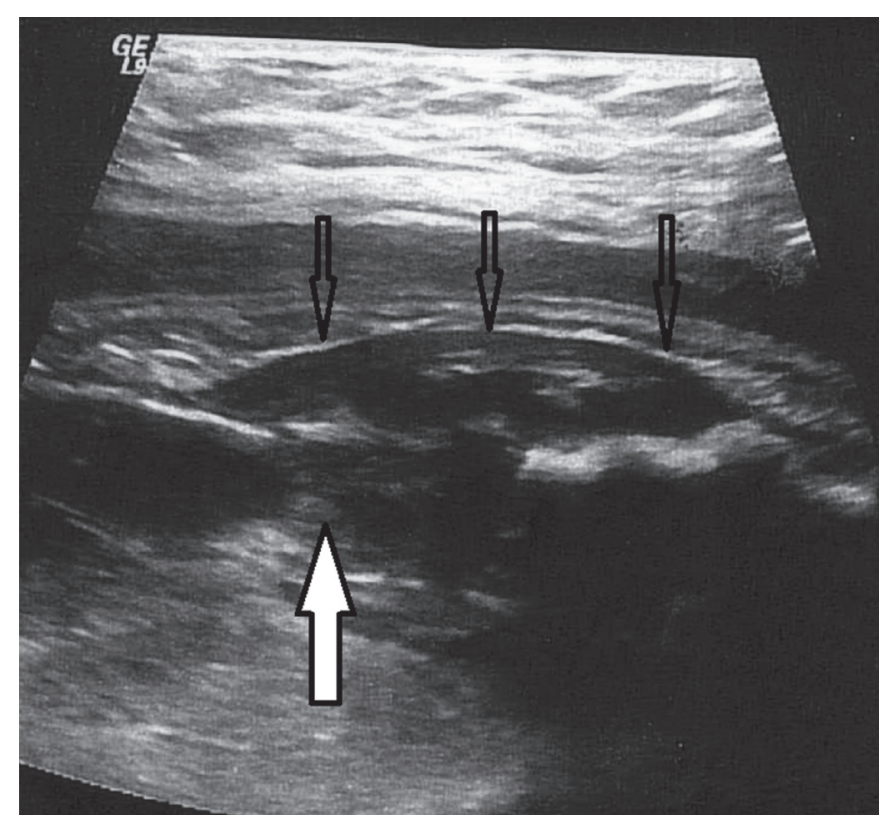

Figure 3: The right mandibular condyle appeared hypoechoic with irregular contour (large white arrow). Fluid retention was seen in the TMJ, and the appearance of the joint capsule was thick and edematous (open arrows).

of mesenchymal cells. Between the $19^{\text {th }}$ and $20^{\text {th }}$ weeks of gestation, typical fibrocartilaginous structures are already evident. ${ }^{4,5}$ After the $25^{\text {th }}$ to $26^{\text {th }}$ weeks of gestation, all of the cellular and synovial components of the TMJ are completely differentiated. The jaw muscles, including the lateral pterygoid, masseter, and temporal muscles were observed during the $9^{\text {th }}$ to $10^{\text {th }}$ gestational weeks; ${ }^{1-4}$ thereby, allowing fetal jaw movement. It is known that forced jaw movements, such as yawning, may cause TMJ dislocation, so fetal jaw movements could be the cause of TMJ dislocation after the $25^{\text {th }}$ to $26^{\text {th }}$ weeks of gestation.

Several hereditary musculoskeletal and connective tissue disorders, such as Ehlers-Danlos syndrome, may involve the TMJ and cause TMJ dislocation. In our case, the father of the fetus had recurrent TMJ dislocation, but there was no history of other joints dislocation or hypermobility that could be compatible with Ehlers Danlos syndrome.

Diagnosis of TMJ disorders is largely based on detailed images; however, a general clinical examination and patient examination should be conducted before scheduling imaging procedures. ${ }^{1}$ Nevertheless, there is insufficient information and research regarding prenatal TMJ ultrasonography; for example, the lengths and masses of condyle or coronoid processes still remain largely unknown. We observed that the most important physical factor for intrauterine TMJ evaluation was fetal position. In our case, the fetus was in the breech presentation; hence, TMJ could be easily evaluated. Although the prenatal diagnosis of TMJ disorders could be important for early treatment, evaluation of the TMJ in the prenatal period may not be necessary during routine sonographic examinations.
In conclusion, antenatal evaluation of the TMJ and diagnosis of the dislocation with real-time sonographic examination is feasible. We presented a case with a developmental disorder of the TMJ, including condylar hypoplasia causing dislocation. TMJ dislocation begins in the prenatal period, and repetitive dislocations may lead to deformation of the joint structure.

\section{References}

1. Axel B, Ulrich L, Mah J. TMJ disorders and orofacial pain: the role of dentistry in a multidisciplinary diagnostic approach. New York: Thieme; 2002.

2. Chhabra S, Chhabra N. Recurrent bilateral TMJ dislocation in a 20-month-old child: a rare case presentation. J Indian Soc Pedod Prev Dent 2011;29:104-106.

3. Cacarini L, Cameron MG. Bilateral TMJ dislocation in a 23-month-old infant: a case report. Dent Update 2009;36:312-313.

4. Lee SK, Kim YS, Oh HS, Yang KH, Kim EC, Chi, JG. Prenatal development of the human mandible. Anat Rec 2001;263:314-325.

5. Berraquero R, Palacios J, Gamallo C, de la Rosa P, Rodriguez JI. Prenatal growth of the human mandibular condylar cartilage. Am J Orthod Dentofacial Orthop 1995;108: 194-200.

\section{Author Affiliations}

Ahmet Said Çil*; Murat Bozkurt'; and Duygu Kara Bozkurt

\section{*Department of Radiology, Universal Hospitals Group, Malatya, Turkey \\ Kafkas University School of Medicine, Department of Obstetrics and Gynecology, Kars, Turkey \\ * Kafkas University School of Medicine, Department of Radiology, Kars, Turkey}

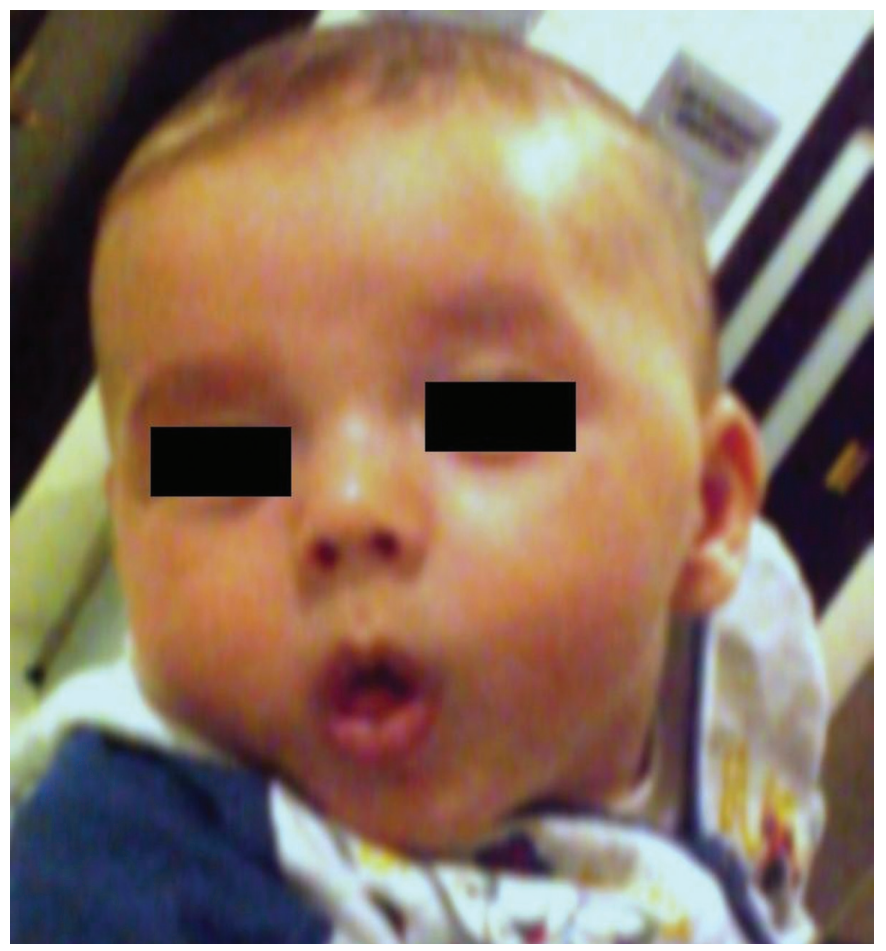

Figure 4: Three months after birth; frontal view of the infant's mouth was opened secondary to malocclusion, and mild facial asymmetry was seen. 